\title{
THE STRUCTURE OF CYCLIC LIE ALGEBRAS
}

\author{
DAVID J. WINTER
}

\begin{abstract}
Simple toral rank 1 Lie algebras have been classified in Wilson [8]. This paper is concerned with the structure of a nonsimple toral rank 1 Lie algebra with respect to a specified "toral rank 1" Cartan subalgebra or, equivalently, with the structure of a nonsimple graded Lie algebra where the grading is the cyclic group grading determined by a specific "toral rank 1" Cartan subalgebra. Such graded Lie algebras are called cyclic Lie algebras, to distinguish them from ungraded toral rank 1 Lie algebras and from graded toral rank 1 Lie algebras where the grading is not a cyclic group grading determined by a "toral rank 1" Cartan subalgebra.

The structure theorems on cyclic Lie algebras of this paper are established by studying $L$ in terms of its graded subalgebras and quotient algebras. Their importance is due to the central role which cyclic Lie algebras play in the theory of Lie algebra rootsystems.
\end{abstract}

1. Introduction. In Kaplansky [4], a simple (finite-dimensional) Lie algebra having a Cartan subalgebra spanned by a single element $h$ such that all roots of ad $h$ lie in the prime field is shown to be $\mathfrak{S l}_{2}$ or the Witt algebra $\mathfrak{W}_{1}$. In Wilson [8], simple Lie algebras of toral rank 1 of characteristic $p>7$ are shown to be $\mathfrak{S l}_{2}$ or in one of the classes $\mathfrak{W}(l: \mathbf{n}), \mathfrak{H}(2: \mathbf{n}: \phi)^{(2)}$. Here, a Lie algebra $L$ is toral rank 1 if $L$ has a cyclic Cartan decomposition $L=\sum_{i=0}^{p-1} L_{i a}$ for some root $a$. In Benkart and Osborn [1], a simple rank one Lie algebra $L$ of characteristic $p>3$ is shown to be $\mathfrak{S l}_{2}$ or Albert-Zassenhaus. Although all of these algebras are, indeed, toral rank one, some Cartan decompositions are not cyclic (except when $L$ is $\mathfrak{S l}_{2}$ or $\mathfrak{W}_{1}$ ), notably those ultimately used for the rank one classification.

In this paper, we study the structure of cyclic Lie algebras, that is toral rank one Lie algebras together with a specified cyclic Cartan decomposition $L=\sum_{i=0}^{p-1} L_{i a}$ over a field of characteristic $p>0$. To distinguish these graded Lie algebras from ungraded toral rank 1 Lie algebras and from graded toral rank 1 Lie algebras where the grading is not a cyclic group grading determined by a "toral rank 1" Cartan subalgebra, we introduce the following terminology. In Definition 1.1, $F\left(L_{0}, k\right)$ denotes the vector space of mappings from $L_{0}$ to $k$.

1.1 Definition. A cyclic Lie algebra is a graded Lie algebra $L=\sum_{g \in G} L_{g}$, where $G$ is an additive cyclic subgroup of $F\left(L_{0}, k\right)$ and

$$
L_{g}=\left\{x \in L \mid(\operatorname{ad} h-g(h) I)^{\operatorname{dim} L} x=0 \text { for all } h \in L_{0}\right\} \quad \text { for } g \in G .
$$

Here, 0 denotes the zero function on $L_{0}$.

Note that the condition on the $L_{g}(g \in G)$ in Definition 1.1 implies that $L_{0}$ is a split Cartan subalgebra of $L$ whose roots are among $0, a, \ldots,(p-1) a$ for any

Received by the editors July 5, 1983 and, in revised form, April 10, 1984 and March 21, 1986.

1980 Mathematics Subject Classification (1985 Revision). Primary 17B05, 17B20, 20H15, 20B25, 20F05; Secondary 05B25. 
generator $a$ for $G$. Thus, $L_{0}$ is a cyclic Cartan subalgebra of $L$ in the sense that $L_{0}$ is a split Cartan subalgebra of $L$ whose roots generate a cyclic group of order 1 or $p$. Conversely, any cyclic Cartan subalgebra $H$ of $L$ in this sense determines, in the form of its Cartan decomposition, a grading for $L$ relative to which $L$ is a cyclic Lie algebra with zero subalgebra $L_{0}=H$.

Cyclic Lie algebras arise in the study of a rootsystem $R$ of an arbitrary Lie algebra $L=\sum_{a \in R} L_{a}$. Specifically, the sections $L a=\sum_{i=0}^{p-1} L_{i a}(a \in R-\{0\})$ of $L$ and the La-modules $L_{b}(a)=\sum_{i=-r}^{q} L_{b+i a}=\sum_{c \in R_{b}(a)} L_{c}(b \in R)$ correspond to sections $R a=R \cap \mathbf{Z} a(a \in R-\{0\})$ and a-orbits $R_{b}(a)(b \in R)$ in $R$. The orbit structure of $R$ is determined by the $L a$-modules $L_{b}(a)(a \in R-\{0\}, b \in R)$. Accordingly, the representation theory for sections $L a(a \in R-\{0\})$ is closely related to the theory of rootsystems $R$ of $L$. Since the choice of $R$ determines a specific grading for the sections $L a(a \in R-\{0\})$, they are studied relative to that grading, that is, they are studied as cyclic Lie algebras in the sense of Definition 1.1

We now state some results used in this paper. Theorem 1.2 is a result on graded Lie algebras more general then cyclic Lie algebras.

1.2 TheOREM (WINTER [10]). A graded Lie algebra $L=\sum_{g \in G} L_{g}$ with $G$ cyclic such that ad $L_{0}$ consists of nilpotent transformations is solvable (nilpotent if $(\operatorname{ad} L)^{p} \subset \operatorname{ad} L$ and all torsion in $G$ is $p^{e}$-torsion $)$.

1.3 TheOREM (WINTER [9]). Let $L$ be a cyclic Lie algebra $L=\sum_{i=0}^{p-1} L_{i a}$. Then the following conditions are equivalent:

(1) $L$ is solvable;

(2) $a\left(\left[L_{i a}, L_{-i a}\right]\right)=0$ for $1 \leq i \leq p-1$;

(3) the ideal $H_{\infty}=L^{\infty} \cap H$ is ad-nilpotent on $L$.

In Theorem 1.3, $L^{\infty}$ is defined as $\bigcap_{i=1}^{\infty} L^{i}$, where $L^{1}=L, L^{2}=[L, L], \ldots, L^{i+1}=$ $\left[L, L^{i}\right], \ldots$ We also define $\operatorname{Solv} L$ as the solvable radical of $L$ and Nil $L$ as the nilpotent radical of $L$.

1.4 THEOREM (SCHENKMAN [5]). Let B be a subnormal subalgebra of $L$. Then $B^{\infty}$ is an ideal of $L$.

1.5 THEOREM (BLOCK [2]). Let $L$ be a differentiably simple Lie algebra. Then $L$ is isomorphic to $S(n)=k[G] \otimes_{k} S$ for some simple Lie algebra $S$ and some integer $n \geq 0$, where $k[G]$ is the group algebra of an elementary abelian $p$ group $G$ of order $p^{n}$. We let $a \mapsto x^{a}$ be an isomorphism from $\mathbf{Z}_{p}^{n}$ to $G$ and denote $G=x^{\mathbf{Z}_{p}^{n}}$.

The following theorem generalizes the corresponding result of Winter [11] from abstract Lie algebras to graded Lie algebras $L$. The proof carries over to the graded case, using Theorem 1 of Winter [10]. In the theorem, Solv $L$ denotes the graded solvable radical of $L$, that is, the (unique) maximal graded solvable ideal of $L$. (Take the trivial grading to recover the theory for abstract Lie algebras.)

1.6 THEOREM. Let $L=\sum_{g \in G} L_{g}$ be a Lie algebra graded by a group $G$ whose torsion is all $p^{e}$-torsion. Then $L$ has a graded subalgebra $L^{\prime}$ such that

(1) $L=L^{\prime}+\operatorname{Solv} L$; 
(2) $L^{\prime} \cap \operatorname{Solv} L$ is nilpotent.

If $H$ is a Cartan subalgebra of $L_{0}$, then $L^{\prime}$ can be taken to be ad $H$-stable.

Let Nil $L$ denote the graded nilpotent radical of a graded Lie algebra $L$, that is, the (unique) maximal graded nilpotent ideal of $L$. The above theorem has the following corollary, wherein a graded Lie algebra $L$ is simple if it has no proper graded ideal; and local if Nil $L$ is a unique maximal graded ideal of $L$.

1.7 COROLLARY. Let $L$ be a Lie algebra graded by a group whose torsion is all $p^{e}$-torsion. Let $L^{\prime}$ be a minimal graded subalgebra such that $L=L^{\prime}+\operatorname{Solv} L$. Then

(1) $L^{\prime} \cap \operatorname{Solv} L$ is nilpotent;

(2) $L^{\prime}$ is local if and only if $L / \operatorname{Solv} L$ is simple.

Proof. By Theorem 1.6, $L^{\prime}$ has a graded subalgebra $L^{\prime \prime}$ such that $L^{\prime}=L^{\prime \prime}+$ Solv $L^{\prime}$ and $L^{\prime \prime} \cap \operatorname{Solv} L^{\prime}$ is nilpotent. It follows that $L=L^{\prime}+\operatorname{Solv} L=L^{\prime \prime}+$ Solv $L^{\prime}+\operatorname{Solv} L$ where $\operatorname{Solv} L^{\prime}+\operatorname{Solv} L$ is a graded solvable ideal of $L=L^{\prime}+\operatorname{Solv} L$. But then Solv $L^{\prime} \subset \operatorname{Solv} L$ and $L=L^{\prime \prime}+\operatorname{Solv} L$. Since $L^{\prime \prime} \subset L^{\prime}$, minimality of $L^{\prime}$ implies that $L^{\prime \prime}=L^{\prime}$. But then Solv $L^{\prime}=L^{\prime \prime} \cap \operatorname{Solv} L^{\prime}$ is nilpotent, that is, Solv $L^{\prime}=\operatorname{Nil} L^{\prime}$. Since $L / \operatorname{Solv} L=L^{\prime} /\left(L^{\prime} \cap \operatorname{Solv} L\right)$ has no nonzero solvable graded ideal, we have Nil $L^{\prime}=\operatorname{Solv} L^{\prime}=L^{\prime} \cap \operatorname{Solv} L$. In particular, $L^{\prime} \cap \operatorname{Solv} L$ must be nilpotent. This proves (1) and establishes that Solv $L^{\prime}=\mathrm{Nil} L^{\prime}$. For one direction of (2), suppose that $L^{\prime}$ is local. We show that $L / \operatorname{Solv} L$ is simple, using the isomorphism $L / \operatorname{Solv} L=L^{\prime} / L^{\prime} \cap \operatorname{Solv} L=L^{\prime} / \mathrm{Nil} L^{\prime}$, by showing that $L^{\prime} / \mathrm{Nil} L^{\prime}$ is simple. In fact, simplicity of $L^{\prime} / \mathrm{Nil} L^{\prime}$ is a direct consequence of the condition that $L^{\prime}$ is local and $L^{\prime} / \mathrm{Nil} L^{\prime}$ is semisimple. For the other direction, suppose that $L / \operatorname{Solv} L=L^{\prime}$ / Nil $L^{\prime}$ is simple. We claim that $L^{\prime}$ is local. Let $I$ be a proper graded ideal of $L^{\prime}$. Then $L \supsetneq I+\operatorname{Solv} L$ by the minimality of $L^{\prime}$ such that $L=L^{\prime}+\operatorname{Solv} L$. Since $L / \operatorname{Solv} L=L^{\prime} /$ Nil $L^{\prime}$ is simple, we conclude that $I \subset L^{\prime} \cap \operatorname{Solv} L=\operatorname{Nil} L^{\prime}$. Thus, $L^{\prime}$ is local.

2. The structure of nonsolvable cyclic Lie algebras. Theorem 1.3 gives the solvability criteria for cyclic Lie algebras. We now turn to the structure of nonsolvable cyclic Lie algebras. We begin with the following version of Schur's Lemma.

2.1 THEOREM. Let $L=\sum_{g \in G} L_{g}$ be a cyclic Lie algebra and let $I$ be an ideal of $L$. Then either $L / I$ is nilpotent or $I$ is solvable (respectively, nilpotent, if $\left.(\operatorname{ad} L)^{p} \subset \operatorname{ad} L\right)$.

Proof. The intersection $I_{0}=I \cap L_{0}$ satisfies either $a\left(I_{0}\right)=\{0\}$ or $a\left(I_{0}\right) \neq\{0\}$, and we have:

(1) $a\left(I_{0}\right)=\{0\}$ if and only if ad $I_{0}$ is nilpotent on $\mathrm{L}$;

(2) $a\left(I_{0}\right) \neq\{0\}$ if and only if ad $I_{0}\left(L_{g}\right)=L_{g}$ for all $g \in G-\{0\}$ (since $g \subset \mathbf{Z}_{p} a$ ).

Here, $a$ is a root generating the cyclic group $G$. If $a\left(I_{0}\right)=\{0\}$ and ad $I_{0}$ is nilpotent on $I$, then $I=\sum_{g \in G} I_{g}$ is solvable (respectively nilpotent, if $(\operatorname{ad} L)^{p} \subset$ ad $L)$, by Theorem 1.2 . If $a\left(I_{0}\right) \neq\{0\}$, then

$$
I \supset[I, L] \supset \sum_{g \in G}\left[I_{0}, L_{g}\right] \supset \sum_{g \in G-\{0\}} L_{g}=L_{*} .
$$


Since $I \supset L_{*}$ and $L=L_{0}+L_{*}, L=L_{0}+I$. But then $L / I=\left(L_{0}+I\right) / I=$ $L_{0} /\left(L_{0} \cap I\right)$. Since $L_{0}$ is nilpotent, $L / I$ is also nilpotent.

Since $B^{\infty}$ is an ideal of $L$ for every subnormal subalgebra $B$ of $L$, by Theorem 1.4, Theorem 2.1 has the following corollary.

2.2 COROLlARY. Every nonsolvable subnormal subalgebra $B$ of a cyclic Lie algebra $L$ contains $L^{\infty}$.

2.3 THEOREM. Let $L=\sum_{g \in G} L_{g}$ be a nonsolvable cyclic Lie algebra. Then

(1) the graded ideal $L^{\infty}$ of $L$ is a cyclic Lie algebra (relative to the grading which it derives from $L$ );

(2) $L^{\infty}=L^{\infty 2}$ and $L^{\infty} / \operatorname{Solv} L^{\infty}$ is a simple cyclic Lie algebra.

Proof. Consider the ideal $I=L^{\infty 2}$ of $L$. By Theorem 2.1, either $L / L^{\infty 2}$ is nilpotent or $L^{\infty 2}$ is solvable. Since $L$ is nonsolvable, it follows that $L / L^{\infty 2}$ is nilpotent. Then we conclude that $L^{\infty} \subset L^{\infty 2}$. The other inclusion clearly also holds. Thus, $L^{\infty 2}=L^{\infty}$. Since $L^{\infty}=H_{\infty}+\sum_{g \in G-\{0\}} L_{g}$ gives the grading $L^{\infty}=\sum_{g \in G} L_{g}^{\infty}$ of $L^{\infty}$, where $L_{0}^{\infty}=H_{\infty}=H \cap L^{\infty}$, showing that $L^{\infty}$ with this grading is cyclic amounts to showing that $L_{0}^{\infty}$ is a Cartan subalgebra of $L^{\infty}$. Since $L^{\infty}$ is nonsolvable, ad $L_{0}^{\infty}$ does not consist solely of nilpotent transformations, by Theorem 1.2. It follows that $a\left(L_{0}^{\infty}\right) \neq\{0\}$, where $a$ is a generator for the cyclic group $G$. Consequently, $\left[L_{0}^{\infty}, L_{g}^{\infty}\right]=L_{g}^{\infty}$ for all $g \neq\{0\}$, which implies that $L_{0}^{\infty}$ is a Cartan subalgebra of $L^{\infty}$. Thus, $L^{\infty}$ is a cyclic Lie algebra. This proves (1). It remains only to show that $L^{\infty} / \operatorname{Solv} L^{\infty}$ is simple, since $\operatorname{Solv} L^{\infty}$ is a graded ideal and $\left(L^{\infty} / \operatorname{Solv} L^{\infty}\right)_{0}=L_{0}^{\infty}+\operatorname{Solv} L^{\infty} / \operatorname{Solv} L^{\infty}$ is a Cartan subalgebra of the graded Lie algebra $L^{\infty} / \operatorname{Solv} L^{\infty}=\sum_{g \in G}\left(L^{\infty} / \operatorname{Solv} L^{\infty}\right)_{g}$ with $\left(L^{\infty} / \operatorname{Solv} L^{\infty}\right)_{g}=$ $L_{g}^{\infty}+\operatorname{Solv} L^{\infty} / \operatorname{Solv} L^{\infty}$. For this, let $I$ be an ideal of $L^{\infty}$ properly containing Solv $L^{\infty}$. We must show that $I=L^{\infty}$. We proved in (1) that $L^{\infty}$ is a cyclic Lie algebra. By Theorem 2.1, we may therefore conclude that either $L^{\infty} / I$ is nilpotent or $I$ is solvable. If $I$ is solvable, then $I=\operatorname{Solv} L^{\infty}$. Thus, we must conclude that $L^{\infty} / I$ is nilpotent. We proved, as the first part of $(2)$, that $L^{\infty}=L^{\infty 2}$. It follows that $L^{\infty}=I$, as asserted by the nilpotency of $L^{\infty} / I$.

We next observe that $\left(L^{\infty}+\operatorname{Solv} L\right) / \operatorname{Solv} L$ is differentiably simple, that is, has no proper ideal invariant under all derivations. For this, consider any ideal $I$ of $L$ contained in $L^{\infty}+\operatorname{Solv} L$ and properly containing Solv $L$. Then $I$ is nonsolvable, so that $I \supset L^{\infty}$ by Theorem 2.1. Since $I \supset \operatorname{Solv} L$, it follows that $I=L^{\infty}+\operatorname{Solv} L$. This shows that $\left(L^{\infty}+\operatorname{Solv} L\right) / \operatorname{Solv} L$ has no proper ideals invariant under the derivations induced by ad $L$, so that $\left(L^{\infty}+\operatorname{Solv} L\right) / \operatorname{Solv} L$ is differentiably simple. We also observe that

$$
\begin{aligned}
\left(L^{\infty}+\operatorname{Solv} L\right) / \operatorname{Solv}\left(L^{\infty}+\operatorname{Solv} L\right) & =\left(L^{\infty}+\operatorname{Solv} L\right) /\left(\operatorname{Solv} L^{\infty}+\operatorname{Solv} L\right) \\
& =L^{\infty} / \operatorname{Solv} L^{\infty}=S,
\end{aligned}
$$

where $S$ is a simple Lie algebra by Theorem 2.3. Since $\left(L^{\infty}+\operatorname{Solv} L\right) / \operatorname{Solv} L$ is differentiably simple and, as just noted, its quotient by its solvable radical is $S$, $L^{\infty}=\operatorname{Solv} L /$ Solv $L$ is isomorphic to $S(n)+k\left[x^{\mathbf{Z}_{p}^{n}}\right] \otimes_{k} S$ for some $n \geq 0$ by Theorem 1.5. This proves the following result.

2.4 TheOREM. Let $L$ be a cyclic Lie algebra. Then $\left(L^{\infty}+\operatorname{Solv} L\right) / \operatorname{Solv} L$ is isomorphic to $k\left[x^{\mathbf{Z}_{p}^{n}}\right] \otimes_{k}\left(L^{\infty} /\right.$ Solv $\left.L^{\infty}\right)$ for some $n \geq 0$. 
When $L$ is restricted, a much stronger version of Theorem 2.3 holds. Recall from $\S 1$ that a graded Lie algebra $L$ is local if $\mathrm{Nil} L$ is the unique maximal graded ideal of $L$. For cyclic Lie algebras, we may drop the adjective "graded" when it applies to ideals, but not when it applies to subalgebras, since the grading is a Cartan decomposition.

2.5 THEOREM. Let $L$ be a restricted cyclic Lie algebra. Then

(1) $L$ is solvable if and only if $L^{\infty}$ is nilpotent;

(2) every nonnilpotent ideal of $L$ contains $L^{\infty}$;

(3) $L^{\infty}$ is a local Lie algebra.

ProOF. For (1), suppose that $L$ is solvable. Then $g\left(\left[L_{g} L_{-g}\right]\right)=0$ for all $g \in G$, by Theorem 1.3. It follows that ad $L_{0}^{\infty}$ consists of nilpotent linear transformations. Since $L$ is restricted, $L^{\infty}$ is therefore nilpotent, by Theorem 1.2. For (2) and (3), we may assume by (1) that $L$ is nonsolvable. For (2), let $I$ be an ideal of $L$ not containing $L^{\infty}$. If $I$ is nonsolvable, the $L / I$ is nilpotent and $L^{\infty} \subset I$, by Theorem 2.1 , a contradiction. Thus, $I$ is solvable. It follows that the $p$-closure $\bar{I}$ of $I$ is solvable. But then $a\left(\bar{I}_{0}\right)=\{0\}$, where $a$ is a generator for the cyclic group $G$, since otherwise $L / \bar{I}$ is nilpotent, as in the proof of Theorem 2.1. It follows that ad $\bar{I}_{0}$ is nilpotent on $L$, hence that $\bar{I}$ is nilpotent, by Theorem 1.2. Thus, $I$ is also nilpotent. For (3), let $I$ be a nonnilpotent ideal of $L^{\infty}$. Then $I$ is an ideal of the $p$-closure $\overline{L^{\infty}}$ of $L^{\infty}$ in $L$. Since $L$ is a cyclic Lie algebra with graded cyclic subalgebra $L^{\infty}, \overline{L^{\infty}}$ is a cyclic Lie algebra with respect to the induced grading. It follows from (2) of this theorem, already established, that $I$ contains $\left(\overline{L^{\infty}}\right)^{\infty}$, since $\overline{L^{\infty}}$ is restricted. But then $I$ contains $L^{\infty}$, that is, $I=L^{\infty}$. Thus, $L^{\infty}$ has no proper nonnilpotent ideals and $L^{\infty}$ is a local Lie algebra.

2.6 COROLlaRY. Let $L=\sum_{a \in R} L_{a}$ be a restricted Lie algebra and let La= $\sum_{i=0}^{p-1} L_{i a}$ for any $a \in R$. Then $(L a)^{\infty}$ is a local cyclic Lie algebra.

PROOF. $L a$ is a restricted subalgebra of $L$, since $(\operatorname{ad} x)^{p} \in \operatorname{ad} L_{0}$ for all $x$ in a basis for $L a$ taken from generating set $\bigcup_{i=0}^{p-1} L_{i a}:(\operatorname{ad} x)^{p}=\operatorname{ad} y,\left[y, L_{0}\right] \subset L_{0+\text { pia }}=$ $L_{0}, y \in L_{0}, \operatorname{ad} y \in \operatorname{ad} L_{0}$.

When $L$ is not restricted, we can prove the following weaker theorem, which still shows that the "simple feature" of any cyclic Lie algebra $L$ can be isolated in a local cyclic graded subalgebra of $L$.

2.7 THEOREM. Let $L$ be a nonsolvable cyclic Lie algebra. Let $L^{\prime}$ be a minimal ad $L_{0}$-invariant subalgebra of $L^{\infty}$ such that $L^{\infty}=L^{\prime}+\operatorname{Solv} L^{\infty}$. Then $L^{\prime}$ is a local cyclic graded subalgebra of $L$ such that $L^{\prime} / \operatorname{Nil} L^{\prime}=L^{\infty} / \operatorname{Solv} L^{\infty}$.

ProOF. We may apply Corollary 1.7 to the graded Lie algebra $L^{\infty}$. The defining condition for $L^{\prime}$ implies that $L^{\prime}$ is a minimal graded subalgebra of $L^{\infty}$ such that $L^{\infty}=L^{\prime}+\operatorname{Solv} L^{\infty}$. Since $L^{\infty} / \operatorname{Solv} L^{\infty}$ is simple, $L^{\prime}$ is a local graded Lie algebra, by Corollary 1.7. Moreover, $L^{\prime}$ is nonsolvable, since $L^{\infty}$ is nonsolvable. It follows from Theorem 1.2 that ad $L_{0}^{\prime}$ does not consist solely of nilpotent elements. Therefore, $L_{0}^{\prime}$ is a Cartan subalgebra of $L^{\prime}$ and $L^{\prime}$, as a graded Lie algebra, is a cyclic graded Lie algebra. Since $L^{\prime}$ is a local graded Lie algebra, and since we may drop the word "graded" for ideals of $L^{\prime}$ since $L^{\prime}$ is a cyclic Lie algebra, $L^{\prime}$ is a local (abstract) Lie algebra. Clearly, $L^{\prime} / \operatorname{Nil} L^{\prime}=L^{\infty} / \operatorname{Solv} L^{\infty}$. 
The above theorem motivates the following definition.

2.8 Definition. Let $L=\sum_{a \in R} L_{a}$ and let $a \in R-\{0\}$. Then a local section of $L$ at $a$ is any minimal ad $L_{0}$-invariant subalgebra $L^{\prime} a$ of $(L a)^{\infty}$ such that $(L a)^{\infty}=$ $L^{\prime} a+\operatorname{Solv}(L a)^{\infty}$.

By Theorem 2.7, the local sections are local cyclic Lie algebras. In passing from a section $L a$ to a local section $L^{\prime} a$, the simple Lie algebra $(L a)^{\infty} / \operatorname{Solv}(L a)^{\infty}$ of the section $L a$ is preserved up to isomorphism as the simple Lie algebra $L^{\prime} a / \mathrm{Nil} L^{\prime} a$. At the same time, the representation theory is considerably simplified by passage from the section $L a$ to the local section $L^{\prime} a$ since the link between the algebra and its associated simple part is much stronger.

Finally, we briefly discuss Wilson's Theorem [8] as it pertains to cyclic Lie algebras, that is, toral rank 1 Lie algebras with a specificed Cartan decomposition. Wilson shows that a simple toral rank one Lie algebra has a maximal subalgebra $L_{(0)}$ whose associated filtration $L_{(-k)} \supset \cdots \supset L_{(-1)} \supset L_{(0)} \supset_{(1)} \cdots$ defined by

$$
\begin{aligned}
& L_{(i+1)}=\left\{x \in L_{(i)} \mid\left[x, L_{(-1)}\right] \supset L_{(i)}\right\} \quad(i \geq 0) \\
& L_{(-i-1)}=\left[L_{(-1)}, L_{(-i)}\right]+L_{(-i)} \quad(i \geq 1)
\end{aligned}
$$

has the following properties:

(1) $L=L_{(-1)}$; and

(2) the zero algebra $L_{(0)} / L_{(1)}$ and its module $L / L_{(0)}$ are one of the following:

(a) $L_{(0)} / L_{(1)}=\mathfrak{G l}_{1}\left(L / L_{(0)}\right)$ with $\operatorname{dim} L / L_{(0)}=1$; or

(b) $L_{(0)} / L_{(1)}=\mathfrak{S l}_{2}\left(L / L_{(0)}\right)$ with $\operatorname{dim} L / L_{(0)}=2$.

Here, $\mathfrak{G l}_{1}\left(L / L_{(0)}\right)$ is $k I$ where $I: L / L_{(0)} \rightarrow L / L_{(0)}$ is the identity map. For a simple cyclic Lie algebra, Wilson's proof shows that there is a maximal graded subalgebra $L_{(0)}$ whose associated gradation $L_{(-k)} \supset \cdots \supset L_{(-1)} \supset L_{(0)} \supset L_{(1)} \supset$ .. has the same properties as before, except that a third possibility must be added under (2):

(c) $L_{(0)} / L_{(1)}=\mathfrak{W}_{1}\left(L / L_{(0)}\right)$ with $\operatorname{dim} L / L_{(0)}=p-1$.

Here, $\mathfrak{W}_{1}\left(L / L_{(0)}\right)$ is the restricted linear Witt algebra of degree $p-1$. Clearly, this result for simple cyclic Lie algebras implies the same result for local cyclic Lie algebras $L$, since $L / \mathrm{Nil} L$ is a simple cyclic Lie algebra with grading inherited from $L$. We now state this result in this general form.

THEOREM (WILSON [8]). Let $L$ be a local cyclic Lie algebra. Then $L / \mathrm{Nil} L$ is isomorphic to one of $\mathfrak{S l}_{2}, \mathfrak{W}(l: \mathbf{n}), \mathfrak{H}(2: \mathbf{n}: \phi)^{(2)}$ and $L$ has a maximal graded subalgebra $L_{(0)}$ whose associated filtration $L_{(-k)} \supset \cdots \supset L_{(-1)} \supset L_{(0)} \supset L_{(1)} \supset \cdots$ has the following properties:

(1) $L=L_{(-1)}$; and

(2) the zero subalgebra $L_{(0)} / L_{(1)}$ and its module $L / L_{(0)}$ are one of the following:

(a) $L_{(0)} / L_{(1)}=\mathfrak{G l}_{1}\left(L / L_{(0)}\right)$ with $\operatorname{dim} L / L_{(0)}=1$; or

(b) $L_{(0)} / L_{(1)}=\mathfrak{S l}_{2}\left(L / L_{(0)}\right)$ with $\operatorname{dim} L / L_{(0)}=2$; or

(c) $L_{(0)} / L_{(1)}=\mathfrak{W}_{1}\left(L / L_{(0)}\right)$ with $\operatorname{dim} L / L_{(0)}=p-1$.

For $L$ simple cyclic, $\bigcap_{i=-1}^{\infty} L_{(i)}=\{0\}$. Therefore, $\bigcap_{i=-1}^{\infty} L_{(i)} \supset$ Nil $L$ for $L$ local cyclic. 


\section{REFERENCES}

1. Georgia Benkart and Marshall Osborn, Rank one Lie algebras, J. Algebra (to appear).

2. Richard Block, Determination of the differentiably simple rings with a minimal ideal, Ann. of Math. (2) 90 (1969), 433-459.

3. Nathan Jacobson, Lie algebras, Interscience, New York, 1962.

4. Irving Kaplansky, Lie algebras of characteristic p, Trans. Amer. Math. Soc. 89 (1958), 149-183.

5. Eugene Schenkman, A theory of subinvariant Lie algebras, Amer. J. Math. 73 (1951), 453-474.

6. George Seligman, Modular Lie algebras, Ergebnisse der Math. Grenzegebiete, Bd. 40, SpringerVerlag, New York, 1967.

7. Robert L. Wilson, The roots of a simple Lie algebra are linear, Bull. Amer. Math. Soc. 82 (1976), 607-608.

8. __ Simple Lie algebras of toral rank one, Trans. Amer. Math. Soc. 236 (1978), 287-295.

9. David J. Winter, Cartgan decompositions and Engel subalgebra triangulability, J. Algebra 62 (1980), 400-417.

10. _ On groups of automorphisms of Lie algebras, J. Algebra 8 (1968), 131-142.

11. __ Reducible complements of Lie algebra radicals, Pacific J. Math. (to appear).

12. __ Symmetric Lie algebras, J. Algebra 97 (1985), 130-165.

Department of MAthematics, University of Michigan, ANn ARbor, Michigan 48109 